\title{
BENCHMARKING AND CONFIGURATION OF OPEN- SOURCE MANUFACTURING EXECUTION SYSTEM (MES) APPLICATION
}

\author{
Ganesha Nur Laksmana ${ }^{1}$; Prianggada Indra Tanaya ${ }^{2}$; Yuki Indrayadi ${ }^{3}$ \\ Department of Industrial Engineering, Swiss German University \\ Edutown BSD City, Tangerang 15339, Indonesia \\ 1'ganesha.laksamana@gmail.com, ² prianggada.itanaya@sgu.ac.id, 3yindraya@gmail.com
}

\begin{abstract}
Information now is an important element to every growing industry in the world. In order to keep up with other competitors, endless improvements in optimizing overall efficiency are needed. There still exist barriers that separate departments in PT. XYZ and cause limitation to the information sharing in the system. Open-Source Manufacturing Execution System (MES) presents as an IT-based application that offers wide variety of customization to eliminate stovepipes by sharing information between departments. Benchmarking is used to choose the best Open-Source MES Application; and Dynamic System Development Method (DSDM) is adopted as this work guideline. As a result, recommendations of the chosen Open-Source MES Application are represented.
\end{abstract}

Keywords: Manufacturing Execution System (MES); Open Source; Dynamic System Development Method (DSDM); Benchmarking; Configuration

\section{INTRODUCTION}

Indonesia is a country with very rich natural resources. Some of the resource sectors that grow rapidly are the mining and forestry sector. Many companies relate to the natural resource; and for the companies, to manage the natural resources of course, heavy equipment is required.

There are currently 2 main heavy equipment companies in Indonesia; one of them is PT. XYZ. With a relatively high competition with the competitor, having the latest technology has become essential to keep up with the competition. One of the technologies widely used is the Enterprise Resource Planning (ERP) which manages and shares information between divisions inside the enterprise to remove every gap inside divisions in the company. Manufacturing Execution System (MES) is a part of ERP. MES focuses only on the production plant. With the latest MES tools, the information sharing inside a manufacturing system will be enhanced which will lead to a higher productivity and efficiency, and in the end, to an increased profit for the company.

\section{Literature Review}

Manufacturing Execution System (MES) is an IT-based tool used in this modern age industries to help managers on the production floor to monitor and record real time activities on the floor.
MES has a relation with Enterprise Information System (EIS), which has the aim to remove stovepipes in the company by information sharing between divisions. This research aims to seek an Open-Source MES by Benchmarking to be configured into PT. XYZ Medium Vessel Assembly Section [1]. The methodology used for this work as the main guideline is the Dynamic System Development Methodology (DSDM). Some experiences gained from implementing DSDM method were also acquired. This result was implemented to maintenance planning and control system [2].

McClellan [3] stated that MES offers benefits as follows: manufacturing cycle time reduced, WIP inventory reduced, paperwork between shifts reduced, lost paperwork/blueprint eliminated, customer service improved, data entry time reduced or even eliminated, lead times reduced, empowered and able to respond unanticipated events.

ISA (International Society of Automation) defined a MES guideline in early 2000 named ISA S95. This guideline is intended to help define and implement MES solution. The main activities or functions of MES based from ISA S95 guideline are: a) Production Resource Management, b) Production Definition Management, c) Detailed Production Scheduling, d) Production Dispatching, 
e) Production Execution Management, f) Production Tracking, g) Production Data Collection and h) Production Performance Analysis.

Since MES is an IT-based application, it has a relation with EIS. EIS means a set of communication in an organization in form of a network that connects and shares information between the components of the organization. In an organization, a department consists of people with similar functionality and is isolated with another department serve as stovepipe. EIS will delete stovepipes inside an organization by sharing information between departments, which will cause the decision-making seen as a whole organization and not as functional areas. An EIS consists of two sides, Model and Architecture. Architecture of EIS has three layers, which are the database layer, application layer and presentation layer. Business process of an enterprise is being modeled in the Model side of EIS. There are many types of model, High level business process model for example Porter's Value Chain, Task Level model for example the Organization Chart, Resource Event Agent (REA) model to see internal or external transactions related with the enterprise, and also Work Flow model to see the steps of work done inside the enterprise in reaching an activity's goal.

In implementing the MES into the system, DSDM is chosen as the main guideline because it is a systematic approach to handle a project effectively and efficiently in form of a framework. DSDM framework consists of five phases: 1) Feasibility Study, 2) Business Study, 3) Functional Model Iteration, 4) Design and Build Iteration and 5) Implementation.

For this research, the design and build phase is replaced by Benchmarking with the assumption that the application is already built and developed. Benchmarking is a comparing activity between two or more application in order to discover the advantages and disadvantages against the similar application so as a result, a decision can be made to choose the better option between the benchmarked applications as stated by Ehliar [4]. Benchmarking is ideal if the application we are interested in is benchmarked with other applications with similar function. The results will not be useful if the intended application is not similar with the benchmarked application. A Benchmark should cover relevant cases to the intended application as the criteria of the Benchmark. It must cover the components, including the object to benchmark with also the core tasks of each component.

\section{METHOD}

The proposed methodology to configure the Open-Source MES Application is the Dynamic System Development Method (DSDM) [5], which consists of five phases.

\section{Feasibility Study}

The first step of DSDM is the feasibility study with the aim to define the objective and scopes as the goal and the coverage of the research. This phase gives clearer understanding of the expectation and constraint of the research by narrowing down the problem areas to cope that fits with the constraints.

\section{Business Study Phase}

Business study phase is the second step of DSDM. This phase aims to obtain information of the current system of the project and to gather users' expectations of the system to be developed. This phase is done by defining the business area, which includes the organization structure, job description, business process model and the expectation of the users and also, making GAP Analysis of the system between the current and expectation.

In business area definition, data collection will be conducted by interviews and direct observations. The result will be analyzed so the current business situation will be defined. This step aims to gain clearer understanding of the current system of the study case, and to identify the expectation for the new proposed system with MES implemented. The results of data collection will be defined in forms of: a) Organizational Structure, which will describe the divisions and agents inside the study case, the relationship between divisions or agents in terms of functionality and also the information flow between them. From organization structure, command line between divisions or agents can be defined and so determining which part of the organization structure will be affected with the implemented MES system. b) Job Description, which will describe tasks of each divisions related with agents inside the study case; c) Business Process Model, which is the workflow model of the system inside the study case. The model describes the flow of information between activities and agents through the system of the study case and d) Users' Expectation. In this step, the statements of users from interviews conducted are being interpreted into users' expectation.

In GAP Analysis, the interpreted users' statement into users' expectation from interview will be used for Benchmarkings between the current system and expected system with proposed solution with MES implemented. The GAP 
Analysis defines the gap between the current and expected condition. The required functions from the users' expectation and gap analysis will be prioritized to get a focus of which requirement will be satisfied first. The prioritization rule to be used is the MoSCoW rule. MoSCoW rule is as follows: 4 = Must Have (Most Important), 3 = Should Have, 2 = Could Have and 1 = Won't Have at present [6]. The result of requirement prioritization will be the highest score of priority will be developed first and then followed by the second highest.

\section{Functional Model Iteration}

After the business study, DSDM continues with functional model iteration phase. The functionality and the expected solution are being modeled in this phase. The models are created based on the business study phase, and must be maintained so the model will fits perfectly with the results of business study phase. The models created are: a) System Interaction Model, which gives a clear picture of the communication between agents and the system inside the study case; b) Resource, Event, Agent (REA) Matrix to define the resource, event and agents in the system. Agents are divided into three categories, initiate, update and read-only. Those 3 are the authorization categories to show different approach of agents to the functions; c) IDEF0 Model, this model used as the companion of REA Matrix. IDEF0 illustrates the transformation input-output of the resources that cannot be seen from the REA Matrix and d) Workflow Model, this is the model of the business process inside the system to describe the flow of information between activities and agents through the system of the study case.

\section{Benchmarking}

The design and build phase will not be taken with the assumption that the MES Application is already developed, but Benchmarking is done to select the best MES Application in replacement of design and build phase. Criteria for Open-Source MES Application Benchmarking include the Database, Programming language, Operating System, Interface, Open-Source License and Price.

\section{Implementation Phase}

In this phase, the MES Application chosen from the Benchmarking will be assessed and then configured to meet the requirements and expectations of the study case based on the functional models created in the earlier phase. The assessment of the MES Application will be using Storyboard Model as reference in analyzing and evaluating the capability of MES application to the business process of the study case. From here, it can be seen if the MES Application can satisfy the requirement or expectation of the case study. The storyboard model will be created directly from the MES Application chosen from the Benchmarking.

Evaluating the Storyboard Model from the early step with the MES Functions as the Business Requirement from the GAP Analysis required for the configuration towards the MES Application. If the MES Functions from the chosen Application does not met with the Requirement or need some tweaks, then configuration with the MES Application chosen will be required in order to satisfy the Requirement.

Further interviews were conducted to determine which of the functions has the highest priority for the system. From the requirement prioritization, it is found that function with the highest priority is the Production Management and Reporting for the Lost or Damage Material and Component. This means the Lost or Damaged Material and Component Problem Reporting Function will be developed first, followed by the second highest priorities.

\section{RESULTS AND DISCUSSION}

The proposed methodology to implement the Open-Source MES Application, which is DSDM, is applied to the Medium Vessel Assembly Section of PT. XYZ [10] [6]. The results of each step of the methodology will be described briefly as follows.

\section{Feasibility Study}

This phase is considered feasible with the acceptance of work proposal from PT. XYZ. The objective of this work is to pick the most suitable Open-Source MES Application by Benchmarking process included in DSDM to fit with the study case. And the scope of this work is related with the area and activities inside the case study, which are gathering the available Open-Source MES Application and analysis of the business process.

\section{Business Study Phase}

The results of business study phase include the core business process, organization structure, users' expectation, GAP analysis, and requirement prioritization. The core business process of PT. $\mathrm{XYZ}$ focuses on production vessels (dump) of heavy equipment vehicles. All the primary activities focus on the production of the vessel and the supporting activities provides the support for the primary activities to perform. From the organization chart, it is known that agents inside the Medium Vessel Assembly Section of PT. XYZ are Supervisor, Foreman, Group Leader and Operator. Figure 1 shows the detailed Organization 
Structure of Medium Vessel Assembly Section of PT. XYZ.

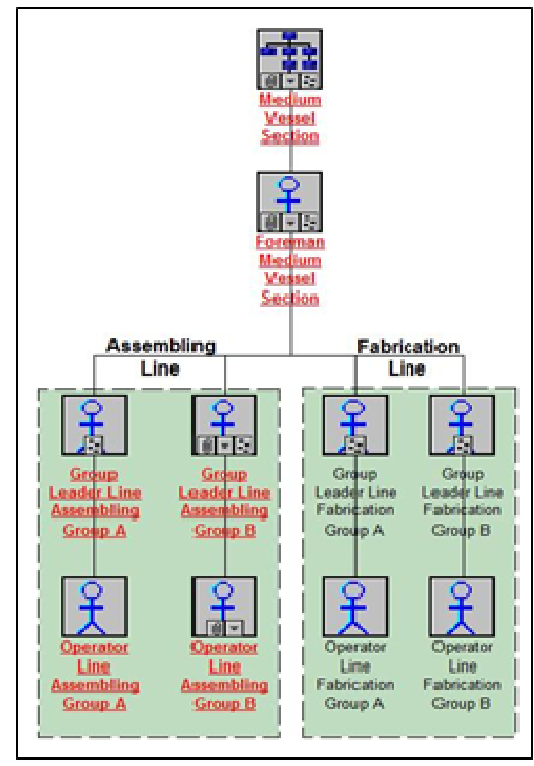

Fig. 1: Organization Structure of Medium Vessel Section [7]

From the interview conducted, users' expectation then interpreted or translated into MES Function Requirement to solve the problem inside the current system. The interpreted needs are: Resource availability can be shown and updated periodically; System is able to serve production and problem reporting to increase information transfer speed; System allows for production progress monitoring; System is able to show the status of product fulfillment; System can serve material picking request required for production to speed up process; System is able to access monthly and daily production schedule; System is able to record finished goods; System can maintain operators' activities reporting; and System is capable of generating painting order.

For the GAP Analysis, the interpreted needs above are interpreted into MES Functions based on the standard of MES by ISA S95: 1) Production Resource Management Function, a) Resource Availability Management, b) Production Resource Request; 2) Detailed Production Scheduling, a) Monthly and Weekly Production Schedule, b) Daily Production Schedule; 3) Production Dispatching, a) Production Work Order Generation; 4) Production Execution Management, a) Production Management and Reporting; 5)Production Tracking, a) Production Operation Monitoring; and 6) Production Performance Analysis, a) Production Performance Status. From the GAP Analysis, it can be found that there are 3 unavailable MES Functions in the study case, with total 8 MES Functions in the study case; it means there are currently 5 MES Functions inside the study case. This means, the implementation of Open-Source MES Application will fill the gap and complete all MES Function required in the study case. Since Open-Source Application means freedom of customization, further developments of the application can be done to meet all the requirements.

Further interviews were conducted to determine which of the functions has the highest priority for the system. From the requirement prioritization, it is found that function with the highest priority is the Production Management and Reporting for the Lost or Damage Material and Component. This means the Lost or Damaged Material and Component Problem Reporting Function will be developed first, followed by the secon highest priorities.

\section{Functional Model Iteration}

In this phase, models of the current system are created and also the models of the proposed solution with the Manufacturing Execution System (MES) Implemented. The models created include System Interaction Model, REA Matrix, IDEF0 Model and Workflow Model.

System Interaction Model, REA Matrix and IDEF0 model mainly describe expected condition with MES Implemented; will be all activities related with the MES Function will be done through the new MES System. While the workflow model describe in more detail how the MES Application is being used in the activities inside the system.

Figure 2 describes the current business process of the current system, and Figure 3 describes the expected workflow after the MES Application is implemented.

\section{Benchmarking}

Benchmarking phase is included in substitution to the design and build iteration phase of DSDM. The result of Benchmarking phase will be the Open-Source MES Application to be implemented into the study case. The first step of Benchmarking phase is the gathering of the OpenSource MES Application. The Open-Source MES Applications gathered are Qcadoo ${ }^{\mathrm{TM}}$, Factority ${ }^{\mathrm{TM}}$, Pinata $^{\mathrm{TM}}$ and MarioMES. The MarioMES is a prototype Open-Source MES Application developed with the same case study (Medium Vessel Assembly Section of PT. XYZ) by Alexander [9] as his work. To increase the variation of the Benchmarking, Open-Source ERP Applications with manufacturing capability are also 
included, since MES is part of ERP. The included Open-Source MES Applications are OpenBravo ${ }^{\mathrm{TM}}$, ADempiere ${ }^{\mathrm{TM}}$ and $\mathrm{xTuple}{ }^{\mathrm{TM}}$.

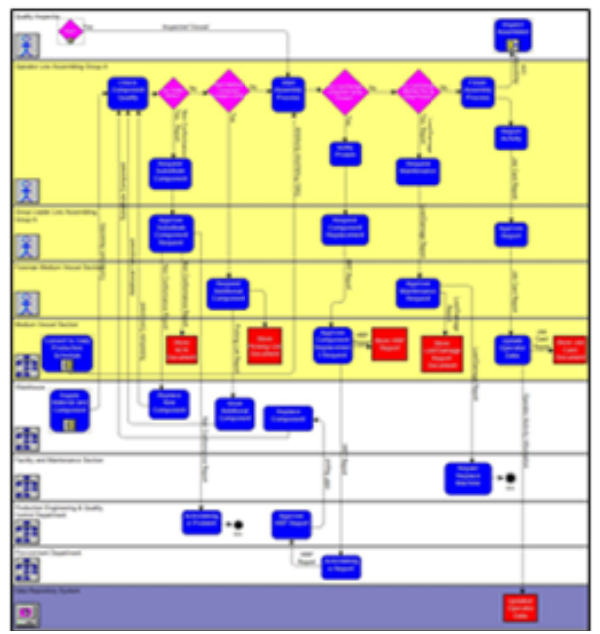

Fig. 2: Workflow of Generic Assembling Process in Medium Vessel Section [7]

The criteria of the Benchmarking include the database, programming language, operating system, interface, open-source license, price, installation, and download size. And from those criteria, Qcadoo ${ }^{\mathrm{TM}}$ is the selected Open-Source MES Application chosen. Qcadoo ${ }^{\mathrm{TM}}$ uses PostgreSQL database which is a free open-source database, withJava programming language that is widely used by program developers, able to run in Windows, MAC and Linux Operating Systems, Qcadoo $^{\mathrm{TM}}$ is a web-based application, the opensource license is Affero General Public License (AGPL), the installation is very easy and it has a relatively small file size with only 32.3Mb.

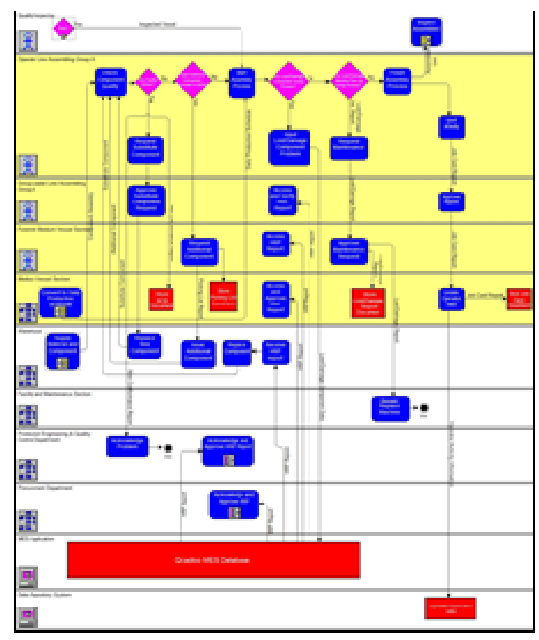

Fig. 3: Proposed Workflow for Generic Assembling Process with MES Implemented [7]
For the price, Qcadoo ${ }^{\mathrm{TM}}$ offers two kinds of products; the QcadooTM Limited is the commercial product web-based Software as a Service (SaaS) with €39/month price and Qcadoo ${ }^{\mathrm{TM}}$ Community Edition which is free for developers to freely develops the Qcadoo ${ }^{\mathrm{TM}}$.

\section{Implementation Phase}

The storyboard of Qcadoo ${ }^{\mathrm{TM}}$ is created in this phase as the assessment of the application. The storyboard model was created based on direct observation of the application. From the storyboard it can be seen the functions in the application to see if the application can fulfill the requirements.

From the storyboard, the MES functions of the applications are defined. From the gap analysis, the business requirements to be satisfied with the MES application functions are also defined. To see which MES function satisfies the requirements and which are not, function conformity matrix is created. To see the agents inside the study case with the MES Functions, function-agent matrix is created.

It is found that from total 8 requirements, Qcadoo $^{\mathrm{TM}}$ only has 6 functions that will satisfy the requirements. This means Qcadoo $^{\mathrm{TM}}$ will have further customization to create new modules to satisfy the remaining 2 requirement.

The configuration of Qcadoo ${ }^{\mathrm{TM}}$ to the study case will include data entry of the available data of PT. XYZ. There are several steps to be done before finally configuring the prioritized MES function. The steps include: a) Business Information, data entry of the basic company data (company name, website, address, etc.); b) Basic Data, data regarding elements inside the system (products, staffs, machines, shifts, and etc.); c) Operation Record, operations or jobs performed inside the facility; d) Technology, operation tree with input and output materials of each operation in order to produce the final product of the business; e) Production Orders, orders received from customer to be processed or production orders to executed.

From the prioritized requirement list, the priority of function do be configured is the Production Management and Reporting for the Lost or Damage Material and Component. Qcadoo ${ }^{\mathrm{TM}}$ offers solution for this requirement with its Stoppages Module. The Stoppages Module defines problem occurred during the production and also the duration delayed caused by the problem occurred.

Accessing the Stoppages Module in Qcadoo $^{\mathrm{TM}}$ can be done in 2 ways, directly from the Production Order to give Stoppages in a specific Production Order like described from the Storyboard model of Order above, or from the 
Stoppages table where all the Stoppages occurred is being shown and also can add new information regarding new stoppage by defining in which Production Order is the Stoppage occurred.

Figure 4 shows the storyboard model of the Stoppages Module from Production orders, and Figure 5 shows the storyboard model of the Stoppages Module from its table.

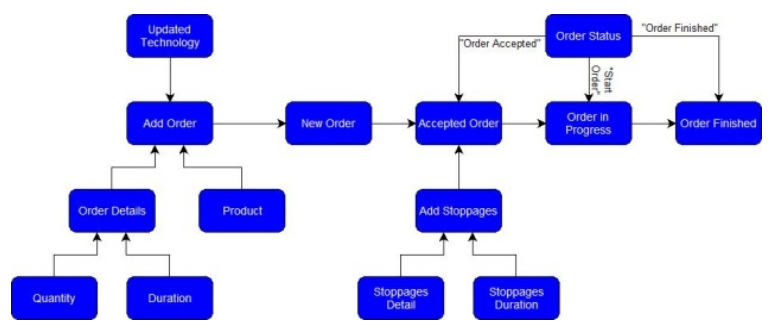

Fig. 4: Qcadoo ${ }^{\mathrm{TM}}$ Storyboard Model: Orders [7]

However, the Stoppages Module in Qcadoo $^{\mathrm{TM}}$ is still general and does not provide a detailed or classified problems occurred. In PT. XYZ Medium Vessel Assembling Section, the Stoppages or Problems in Production are divided into three types, Lost/Damage Material/Component, Material Defect and Lost/Damage Machine part/jig. This means a further development in the Stoppages Module of Qcadoo $^{\mathrm{TM}}$ is needed to provide a classified problem in the Stoppage Module to provide more accurate information.

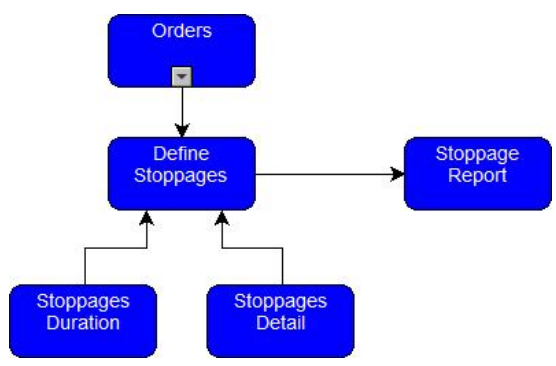

Fig. 5: Qcadoo ${ }^{\mathrm{TM}}$ Storyboard Model: Stoppages [7]

\section{CONCLUSION}

The information flow in Medium Vessel Assembly Section of PT. XYZ is still not optimum. From the gap analysis, the current system still does not satisfy the entire business requirement. OpenSource MES Application offers the solution to satisfy all the business requirements in the study case. Open-Source means the application is free to customize or develop more modules to meet the requirements. Benchmarking is used to choose the best Open-Source MES Application available.

Based on the result of this work, Qcadoo ${ }^{\mathrm{TM}}$ is chosen as the better Open-Source MES
Application. Qcadoo ${ }^{\mathrm{TM}}$ has been assessed and configured during this work. It is found that currently Qcadoo $^{\mathrm{TM}}$ can only satisfy 6 (75\%) out of 8 business requirements in PT. XYZ Medium Vessel Assembly Section.

In Conclusion, further developments (develop new modules) are needed for the Qcadoo $^{\mathrm{TM}}$ to satisfy all the business requirements in PT. XYZ Medium Vessel Assembly Section.

\section{REFERENCES}

[1] C. Thenario, A Case Study of Functional Model Definition For Manufacturing Execution System Prototype Development In The Assembly Line Of Medium Vessel Section By Using Dynamic System Development Method, Tangerang: Department of Industrial Engineering, Swiss German University, 2011.

[2] G. Wicaksono, A Case Study of CMMS Application Configuration For Base Maintenance Planning And Control Unit By Using Dynamic System Development Method, Tangerang: Department of Industrial Engineering, Swiss German University, 2011.

[3] M. McClellan, "Introduction to Manufacturing Execution System”, in Jun 2000 Mes Conference \& Exposition, Available:

http://ime.syr.edu/PDF\%20files/Intro_MES_McClellan.pdf

[4] A. Ehliar and D. Liu, Benchmarking Network Processors, 2004, Available: http://www.da.isy.liu.se/pubs/ehliar/ehliarssocc2004.pdf

[5] P. K. Gorakavi, Build Your Project Using Dynamic System Development Method \#5 of a Series, Dec. 2009. Available: http://www.asapm.org/asapmag/articles/A5_AboutDSDM.p df

[6] Coley Consulting, MoSCoW Prioritization, Available: http://www.coleyconsulting.co.uk/moscow.htm

[7] G. N. Laksmana, Benchmarking and Configuration of Open-Source Manufacturing Execution System (MES) Application, Tangerang: Department of Industrial Engineering, Swiss German University, 2012.

[8] D. Clark, ISA S95, 2010, Available: http://iom.invensys.com/EN/pdfLibrary/Newsletter/Presenta tion_ISA\%20S95.ppt

[9] M. Alexander, Manufacturing Execution System Application Prototype Development Using Dynamic System Development Method, Tangerang: Department of Industrial Engineering, Swiss German University, 2011.

[10] C. L. Dunn, J. O. Cherrington, and A. S. Hollander, Enterprise Information Systems: A Pattern-Based Approach, 3rd Edition, New York: McGraw-Hill, 2005. 